Research Article

\title{
Chitosan-S-triazinyl-bis(2-aminomethylpyridine) and Chitosan- $S$-triazinyl-bis(8-oxyquinoline) Derivatives: New Reagents for Silver Nanoparticle Preparation and Their Effect of Antimicrobial Evaluation
}

\author{
Hessa H. Al-Rasheed $\mathbb{D},{ }^{1}$ Kholood A. Dahlous, ${ }^{1}$ Essam N. Sholkamy $\mathbb{D}^{,},{ }^{2}$ Sameh M. Osman, \\ Omar H. Abd-Elkader, ${ }^{3,4}$ and Ayman El-Faham $\mathbb{D}^{1,5}$ \\ ${ }^{1}$ Chemistry Department, College of Science, King Saud University, P.O. Box 2455, Riyadh 11451, Saudi Arabia \\ ${ }^{2}$ Department of Botany and Microbiology, College of Science, King Saud University, P.O. Box 2455, Riyadh 11451, Saudi Arabia \\ ${ }^{3}$ Physics and Astronomy Department, College of Science, King Saud University, P.O. Box 2455, Riyadh 11451, Saudi Arabia \\ ${ }^{4}$ Electron Microscope and Thin Films Department, Physics Division, National Research Center (NRC), El-Behooth Street, Dokki, \\ Cairo 12622, Egypt \\ ${ }^{5}$ Department of Chemistry, Faculty of Science, Alexandria University, P.O. Box 426, Ibrahimia, Alexandria 21321, Egypt
}

Correspondence should be addressed to Hessa H. Al-Rasheed; halbahli@ksu.edu.sa and Ayman El-Faham; aymanel_faham@ hotmail.com

Received 15 December 2019; Revised 29 April 2020; Accepted 25 May 2020; Published 25 June 2020

Academic Editor: Long Bai

Copyright (c) 2020 Hessa H. Al-Rasheed et al. This is an open access article distributed under the Creative Commons Attribution License, which permits unrestricted use, distribution, and reproduction in any medium, provided the original work is properly cited.

\begin{abstract}
Herein, we described the modification of chitosan with cyanuric chloride as a mediator for preparation of chitosan-s-triazinylbis(2-aminomethylpyridine) and chitosan-s-triazinyl-bis(8-oxyquinoline) derivatives to be used as reagents for preparation of silver nanoparticles under ecofriendly conditions. These two reagents are convenient and effective for reduction of silver ions to silver nanoparticles with particle size less than $10 \mathrm{~nm}$ that might be suitable for industrial and medicinal applications. The formation and particle size of AgNPs are characterized by transmission electron microscopy (TEM), X-ray diffraction (XRD), scanning electron microscope (SEM), and energy-dispersive X-ray analysis (EDX). The antimicrobial activity of the two modified chitosan-s-triazine-AgNPs was evaluated against activities against Gram-positive bacteria (M. luteus ATCC 10240 and MRSA ATCC 43300), Gram-negative bacteria (E. coli ATCC 25922 and P. aeruginosa ATCC 75853), and C. albicans. The results showed that chitosan-s-triazinyl-bis(2-aminomethylpyridine) AgNPs showed high antimicrobial activities against all the tested microorganisms, while their analogous chitosan-s-triazinyl-bis(8-oxyquinoline) AgNPs showed moderate activities.
\end{abstract}

\section{Introduction}

In recent years, efficacy of nanoparticles has been the subject of great interest due to their unique physical and chemical properties, which make them significantly different from their counterpart in the bulk form [1-5]. In particular, silver nanoparticles have gained special interest in recent years because of their outstanding physical, chemical, and biological properties [6-11]. Several methods were used for the synthesis of AgNPs [12-17]; among them, the chemical reduction methods were considered as the most common methods used for the synthesis of AgNPs. Generally, the reduction of silver ions in the chemical methods occurred in the presence of a reducing agent such as hydrazine, ammonium formate, and sodium borohydride [17-22]. These reducing agents are somewhat considered as potentially dangerous to the environment and biological system. Numerous methods have been reported for the synthesis of AgNPs using green, cost-effective, and biocompatible, among them is using extracts of medicinal plants [23-26]. 
On the contrary, chitosan has excellent biodegradability, antibacterial effect, hemostatic action, and anti-inflammatory effect. Because of these properties, it gains extensive use in pharmaceutical, food, biotechnology, nanobiocatalyst, and agricultural applications [9, 27-34], eliminating heavy minerals, phosphorus, and oil from water [35-39]. There are several publications regarding chitosan modifications through amino groups. However, chemical modifications in the fundamental skeleton of chitosan may lose its original physicochemical and biochemical activities [40-43].

Recently, we reported the synthesis of 2,4,6-tris(quinolin-8-yloxy)-1,3,5-triazine and $N^{2}, N^{4}, N^{6}$-tris(pyridin-2ylmethyl)-1,3,5-triazine-2,4,6-triamine; these derivatives showed promising organic corrosion inhibitors for steel in $\mathrm{HCl}$ solutions [44]. Accordingly, herein, we describe the modification of chitosan with cyanuric chloride as a mediator for preparation of substituted $s$-triazine with two armed symmetrical group 2-aminopyridine or 8-oxyquinoline. This new modification proved to be convenient and effective for the reduction of silver nitrate to silver nanoparticles under ecofriendly conditions (water medium) without using any reducing or stabilizing agent. In addition, the antimicrobial activities of the modified chitosan-s-triazine AgNPs were evaluated as well.

\section{Experimental Section}

2.1. Materials and Methods. Chitosan (medium molecular weight) and 2,4,6-trichloro-1,3,5-triazine (TCT) were purchased from Sigma-Aldrich, and silver nitrate was purchased from Merck. All the chemicals and solvents used were analytical graded and used without further purification. Thermal properties of chitosan and modified chitosan were examined using Mettler Toledo model TGA/DSC1 (Mettler Toledo Ltd., Boston Road, Leicester, UK). The electronic spectra of AgNPs were measured using UV-Vis spectroscopy (Perkin Elmer Lambda 35; Waltman, MA, USA). The evaluation of the crystal structure was achieved by using the X-ray diffractometer (XRD) (X'Pert PRO, PANalytical BV, Almelo, the Netherlands) using $\mathrm{Cu} \mathrm{K}_{\alpha}$ radiation. The morphology, composition, and particle size of AgNPs were performed by means of the scanning electron microscope (SEM) equipped with energy-dispersive spectrometry analysis (EDS) (JEOL SEM 6380 LA, Japan) and transmission electron microscopy (TEM) (JEOL JEM 1010, Japan). Samples for TEM studies were prepared by placing drops of AgNP solutions on the carbon-coated, and the histograms of AgNPs' size distribution were calculated from the TEM images.

2.2. Synthesis of Chitosan-4,6-dichloro-1,3,5-triazine (3). Synthesis of chitosan-4,6-dichloro-1,3,5-triazine was carried out by modification of the reported method [27] as follows: chitosan $(20.0 \mathrm{gm})$ was suspended in toluene $(200 \mathrm{~mL})$ followed by the slow addition of cyanuric chloride 1 (27.60 gm; $150 \mathrm{mmol})$ and sodium carbonate $(16.0 \mathrm{gm}, 150 \mathrm{mmol})$ at room temperature with vigorous stirring. The reaction temperature was raised gradually to $70-80^{\circ} \mathrm{C}$ and kept at this temperature for 24 hours (ninhydrin test was negative as shown in Figure S1, Supplementary Materials). The solid product was separated by filtration, washed with toluene and mixture of waterethanol $(1: 1)$, and then dried at $50^{\circ} \mathrm{C}$ for 6 hours to afford modified chitosan Ch-TC2, 3.

2.3. Synthesis of Chitosan-4,6-disubstituted-1,3,5-triazine, 6 and 7. 8-Hydroxyquinoline 4 or 2-aminomethylpyridine 5 (100 mmol) was added to a suspended solution of $3(5.0 \mathrm{gm})$ in tetrahydrofuran (THF, $50 \mathrm{~mL}$ ) followed by addition of triethylamine $(200 \mathrm{mmol})$. The reaction mixture was refluxed overnight, and the solid product was separated by filtration washed with dichloromethane and ethanol, and then dried at $60^{\circ} \mathrm{C}$ for 12 hours to afford the target products Ch-TQ 6 and Ch-TAMPy 7 in almost quantitative yields.

2.4. Synthesis of Silver Nanoparticles (AgNPs). Modified chitosan ( $0.1 \mathrm{gm}, \mathrm{Ch}$-TQ or Ch-TAMPy) was suspended in $50 \mathrm{~mL}$ water, and then solution of $\mathrm{AgNO}_{3}$ in water $(50 \mathrm{~mL}$, $10^{-3} \mathrm{M}$ ) was added dropwise with stirring at room temperature; after complete addition, the mixture was kept overnight at room temperature. Color of the solution was changed from colorless to brownish black with no further color observed for longer time (Figure S2, Supporting Information). The AgNPs immobilized on modified chitosan were collected by centrifugation at $8000 \mathrm{rpm}$ for 5 minutes, then washed with water, and dried at $60^{\circ} \mathrm{C}$ for 12 hours.

2.5. Characterization of Silver Nanoparticles ( $U V$-Vis, XRD, $S E M$, and TEM). UV-Vis analysis was performed in quartz cuvettes using double distilled water as a reference solvent, and scanning was performed after the reaction was completed in the range $250-870 \mathrm{~nm}$ absorbance spectrum in steps of $1 \mathrm{~nm}$. XRD analysis was performed at $40 \mathrm{kV}$ and $40 \mathrm{~mA}$. The scans were typically performed over a $2 \theta$ range from $10^{\circ}$ to $85^{\circ}$ at a scan rate of $0.02 \_/ s$, with an aperture slit, an antiscatter slit, and a receiving slit of $2 \mathrm{~mm}, 6 \mathrm{~mm}$, and $0.2 \mathrm{~mm}$, respectively. X-ray $\mathrm{Cu} \mathrm{K} \alpha$ wavelength was $1.54056 \AA$. The surface morphology was examined using the scanning electron microscope (SEM). The elemental compositions of AgNPs were measured using the high-energy beam of electrons. X-rays were detected using an energydispersive spectrometry analyzer (EDS) combined with SEM. The formation of nanoparticles was confirmed by using compact high-performance transmission electron microscopy (TEM).

2.6. Thermogravimetric Analysis (TGA) for Ch-TQ and ChTAMP $y$. Thermal properties of modified chitosan (Ch-TQ and Ch-TAMPy) were examined using TA-Q500, and the data were collected under nitrogen with a flow rate of $60 \mathrm{~mL} /$ min in the temperature range $25^{\circ} \mathrm{C}-800^{\circ} \mathrm{C}$ with a different heating rate of $5,10,20$, and $25^{\circ} \mathrm{C} / \mathrm{min}$. 
2.7. Antibacterial Activity. Antibacterial activities of two modified chitosan-AgNPs (Ch-TQ 6 and Ch-TAMPy 7) against the test organisms, E. coli ATCC 25922, P. aeruginosa ATCC 75853, M. luteus ATCC 10240, MRSA ATCC 43300, and C. albicans ATCC 10145, were determined by the modified Kirby-Bauer disk diffusion method [45] (as described in Method S1, Supplementary Materials).

\section{Results and Discussion}

Chitosan was modified by using cyanuric chloride (TCT, $\mathbf{1}$ ) in the presence of $\mathrm{Na}_{2} \mathrm{CO}_{3}$ as the $\mathrm{HCl}$ scavenger to ensure complete capping for the amino groups in the chitosan skeleton as observed from the ninhydrin test (Figure S1, Supporting Information). Modified chitosan 3 was reacted with 8-hydroxyquinolne 4 or 2-aminomethylpyridine 5 in the presence of triethylamine to afford Ch-TQ 6 and ChTAMPy 7 as shown in Scheme 1.

Figure 1 exhibits the TGA degradation curves of $\mathrm{Ch}, \mathrm{Ch}$ TQ, and Ch-TAMPy. The thermal degradation parameters are summarized in Table S1 (Supporting Information). The results showed that modified chitosan (Ch-TQ and ChTAMPy) have slight change in the thermal stability with two main degradation steps. In case of chitosan, the first step starts in a range of $36^{\circ} \mathrm{C}$ to $167^{\circ} \mathrm{C}$, with mass loss of $7.7 \%$. However, in case of modified chitosan, the first step starts in a range of $34^{\circ} \mathrm{C}$ to $167^{\circ} \mathrm{C}$ with mass loss of $7.8 \%$ and in a range $23^{\circ} \mathrm{C}$ to $187^{\circ} \mathrm{C}$ with mass loss of $7.8 \%$ and $9.5 \%$ for ChTQ and Ch-TAMPy, respectively, which could be due to evaporation of the solvent content. The second degradation step starts in a range of $290^{\circ} \mathrm{C}$ to $450^{\circ} \mathrm{C}$, with mass loss of $49.3 \%$ in case of chitosan, while in case of modified chitosan, the second step starts in a range of $287^{\circ} \mathrm{C}$ to $449^{\circ} \mathrm{C}$ with mass loss of $49.3 \%$ and in a range of $255^{\circ} \mathrm{C}$ to $425^{\circ} \mathrm{C}$ with mass loss of $29.3 \%$ and $39.8 \%$ for Ch-TQ and Ch-TAMPy, respectively.

Figures 2(a)-2(c) represent the SEM micrographs illustrating the morphology of $\mathrm{Ch}, \mathrm{Ch}-\mathrm{TQ}$, and Ch-TAMPy. The SEM micrograph of pure chitosan (Figure 2(a)) showed a smooth surface, while both modified chitosan 6 and 7 (Figures 2(b) and 2(c)) showed very rough surface morphology compared with chitosan itself.

Modified chitosan 6 and 7 were used for preparation of AgNPs in water; the color change in the reaction mixture was the initial indicator of the formation of AgNPs (Figure S2, Supplementary Materials). The formation of dark brown color arises from the excitation of the AgNP plasmon [46].

The UV-Vis absorption spectra (Figure S3, Supplementary Materials) for the solution obtained from Ch-TQAgNPs and Ch-TAMPy-AgNPs showed absorption bands near $420 \mathrm{~nm}$ related to the silver surface plasmon. This indicates complete conversion of silver ions $(\mathrm{Ag}+)$ to $\mathrm{Ag}^{\mathrm{O}}$ (AgNPs) [47]. The AgNPs formed by using two modified chitosan 6 and 7 were found to be very stable for 5-6 months; this might be due to the presence of the hydroxyl group in the chitosan skeleton which prevents agglomeration of AgNPs [48-50].

EDS analysis established the presence of the elementary silver signal of the prepared AgNPs (Figures S4A and S4B, Supporting Information), where strong signal peaks in the range of 2.5-4 keV were observed; these peaks correspond to the binding energies of crystalline silver [51]. A strong signal peak near $0.2 \mathrm{keV}$ was observed which is corresponding to carbon in the ligand connected to AgNPs.

The XRD patterns (Figures 3(a) and 3(b)) showed peaks at about $2 \theta=38.10^{\circ}, 44.09^{\circ}, 64.36^{\circ}, 77.29^{\circ}$, and $81.31^{\circ}$ for both prepared modified chitosan; these peaks correspond to 111 , $200,220,311$, and 420 planes, respectively. This indicates a typical face-centered cubic structure of silver as per the available literature (Powder Diffraction File, PDF file no. 04-0783) [52, 53].

The lattice parameters, unit cell volume, and space group were calculated with the help of the FullProf and Chekcell programs $[R]$. The observed and calculated crystallographic data Miller indices $(h k l), d$ spacing (the interplanar spacing), and $2 \theta$ (the diffraction angle) were also calculated and are matched with the standard values of Ag [53]. The average grain size $(D)$, dislocation density $(\delta)$, and the strain $(\varepsilon)$ of the AgNPs are calculated, and the average grain size $(D)$ for both samples is found to be in the range $40-45 \mathrm{~nm}$ (Figure S5, Tables S2-S4, Supplementary Information).

The SEM (Figure 4) for samples from Ch-TQ-AgNPs and Ch-TAMPy-AgNPs showed a uniform structure and a homogenous distribution of AgNPs over the modified chitosan surface as shown in Figures 4(a) and 4(b).

The TEM image of AgNPs formed by using $\mathbf{6}$ and 7 is represented in Figures 5(a) and 5(b), respectively. Analysis of TEM imaging showed that prepared AgNPs are monodispersed spheres in the range of $2-10 \mathrm{~nm}$ in size for both samples prepared by using 6 and 7 . AgNPs were formed after reduction by the lone pair of electrons of the nitrogen atom at 2-aminomethypyrindine and 8-hydroxyquinolone side chains and triazine moiety in the ligand structure. Particles with diameters more than $10 \mathrm{~nm}$ were formed due to aggregation through preparation of the TEM holding grid.

Finally, both synthesized AgNPs from Ch-TQ and ChTAMPy exhibit good antimicrobial activity against all the test organisms in the Kirby-Bauer assay. The diameters of the zone of inhibitions produced by these compounds against Gram-positive bacteria (M. luteus and MRSA), Gram-negative (E. coli and $P$. aeruginosa) bacteria, and against $C$. albicans with various concentrations of the test compounds are listed in Table 1. It is interesting to note that these zones of inhibitions were obtained even with very low concentration $(10-40 \mu \mathrm{g} / \mathrm{well})$ of the test compounds. Chitosan derivatives are used as the control and did not show any bioactivity against the tested pathogenic microbes, while the synthetic silver nanoparticles have bioactivity against the tested microbes. The data presented in Table 1 showed that Ch-TAMPy-AgNPs exhibited higher antimicrobial activities against the tested microorganisms specially at higher 
<smiles>Clc1nc(Cl)nc(Cl)n1</smiles>

TCT, 1

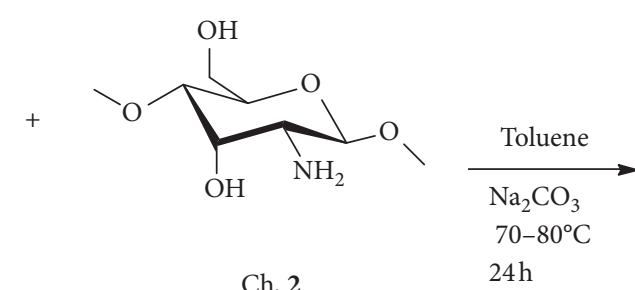

Ch, 2<smiles></smiles><smiles>C=C(Cl)N=C(C)Cl</smiles>

Ch-TC2; 3

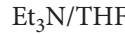
reflux $24 \mathrm{~h}$<smiles>Oc1cccc2cccnc12</smiles>

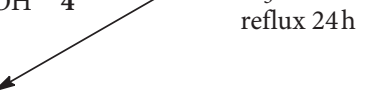

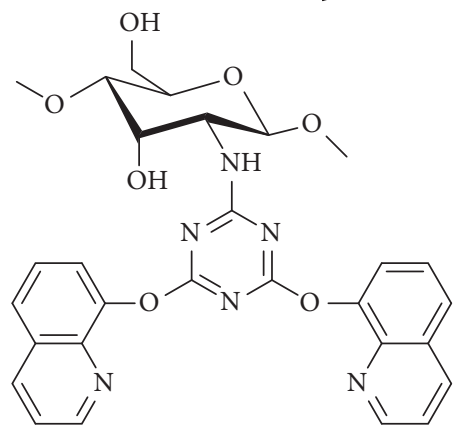

Ch-TQ; 6<smiles>NCc1ccccn1</smiles>

Scheme 1: Synthesis of modified chitosan Ch-TQ 6 and Ch-TAMPy 7.

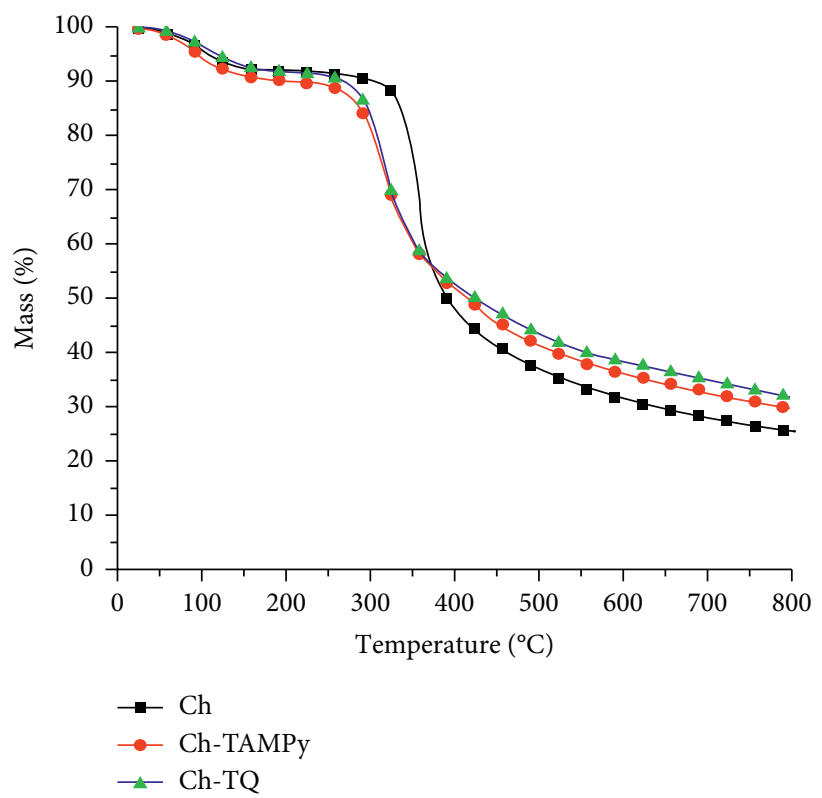

Figure 1: TGA degradation curves for $\mathrm{Ch}, \mathrm{Ch}-\mathrm{TQ}$, and Ch-TAMPy.

concentration $(30 \mu \mathrm{g} /$ well and $40 \mu \mathrm{g} /$ well) than Ch-TQAgNPs, which is in agreement with the reported data for pyridine derivatives $[54,55]$.

In addition, as shown in Table 1, Gram-negative bacteria were found to be less sensitive than Gram-positive; this may be due to the outer membrane components of Gram-negative bacteria, which may absorb the AgNPs and prevent them from penetration of the bacterial cell wall. This is also in agreement with the finding report on silver nanoparticles synthesized using Pulicaria glutinosa plant extracts [56]. 


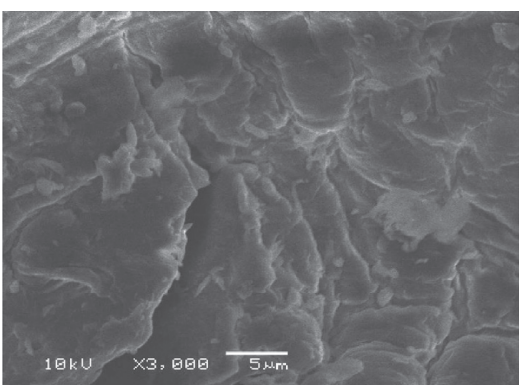

(a)

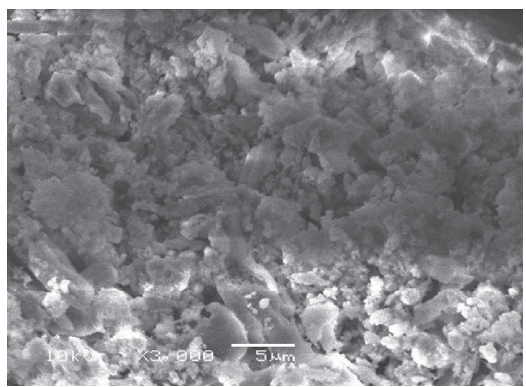

(b)

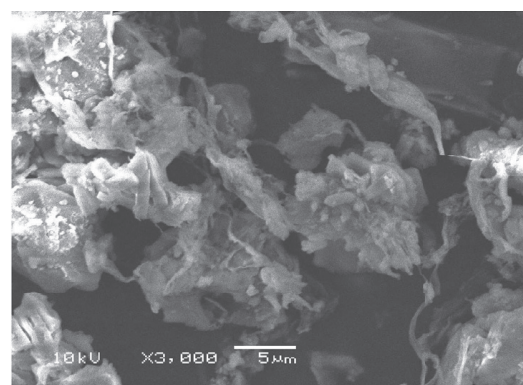

(c)

Figure 2: SEM images of chitosan/modified chitosan: (a) chitosan; (b) Ch-TQ; (c) Ch-TAMPy.

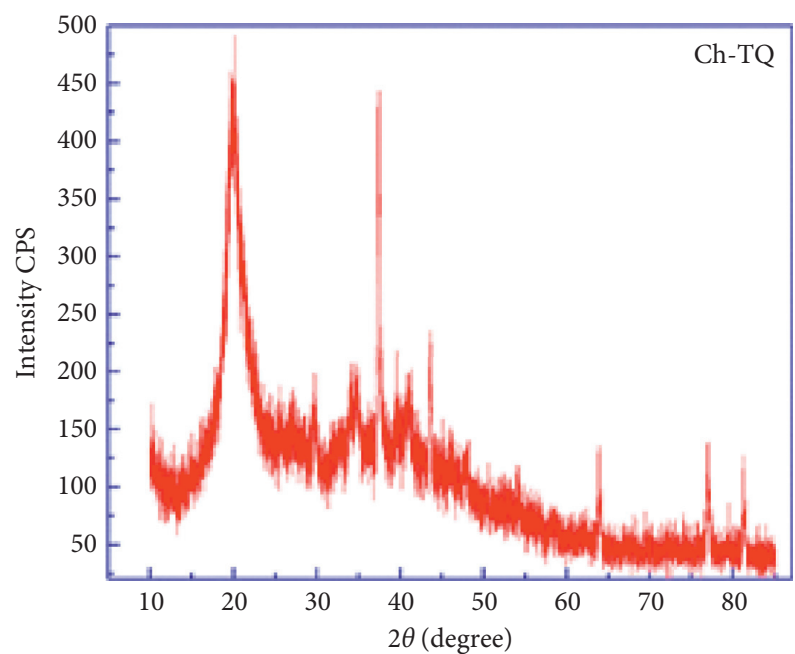

(a)

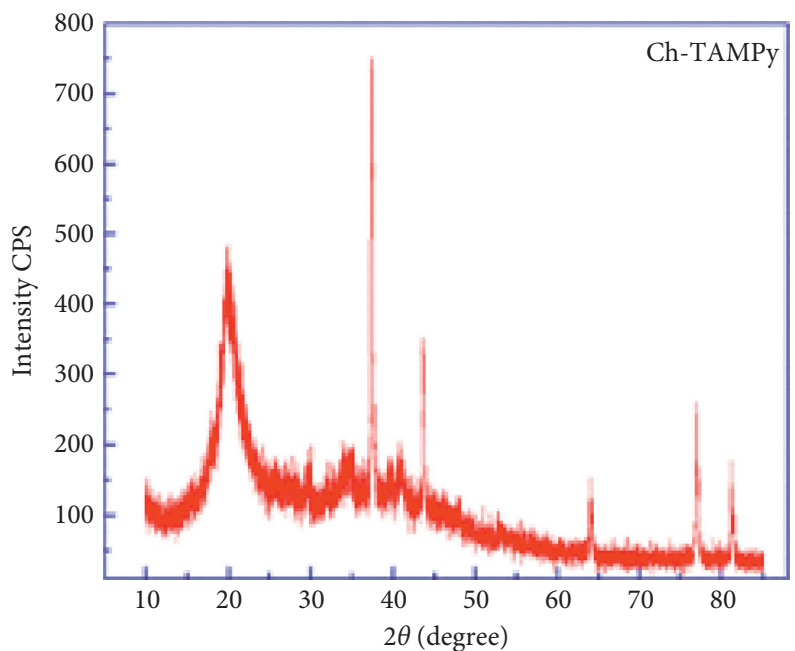

(b)

Figure 3: (a) X-ray diffraction pattern of the silver nanoparticles produced by employing Ch-TQ; (b) X-ray diffraction pattern of the silver nanoparticles produced by employing Ch-TAMPy.

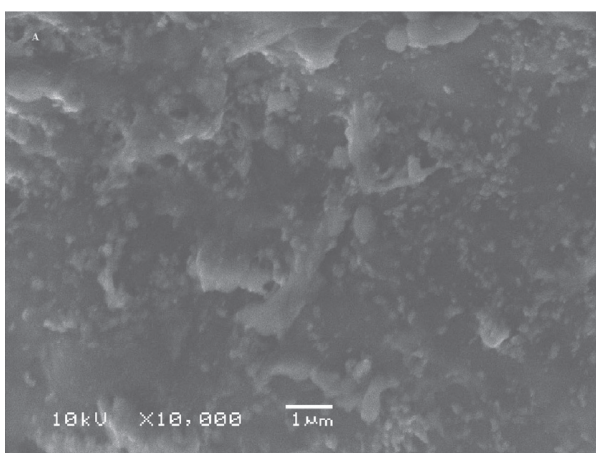

(a)

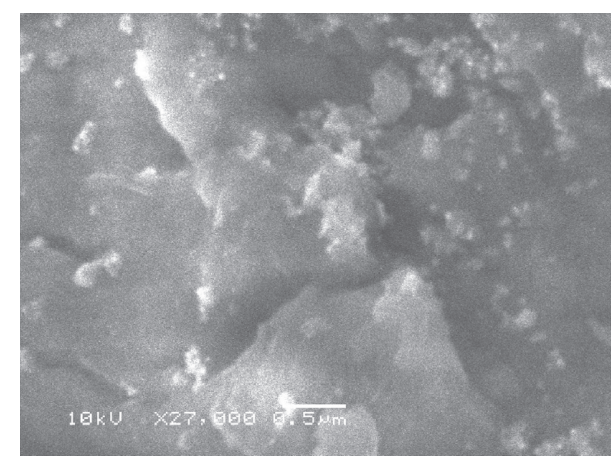

(b)

FIGURE 4: High-resolution electron microscope (SEM) micrograph: (a) silver-Ch-TQ composite using the secondary electron imaging mode (SEI); (b) silver-Ch-TAMPy composite using the secondary electron imaging mode (SEI). 


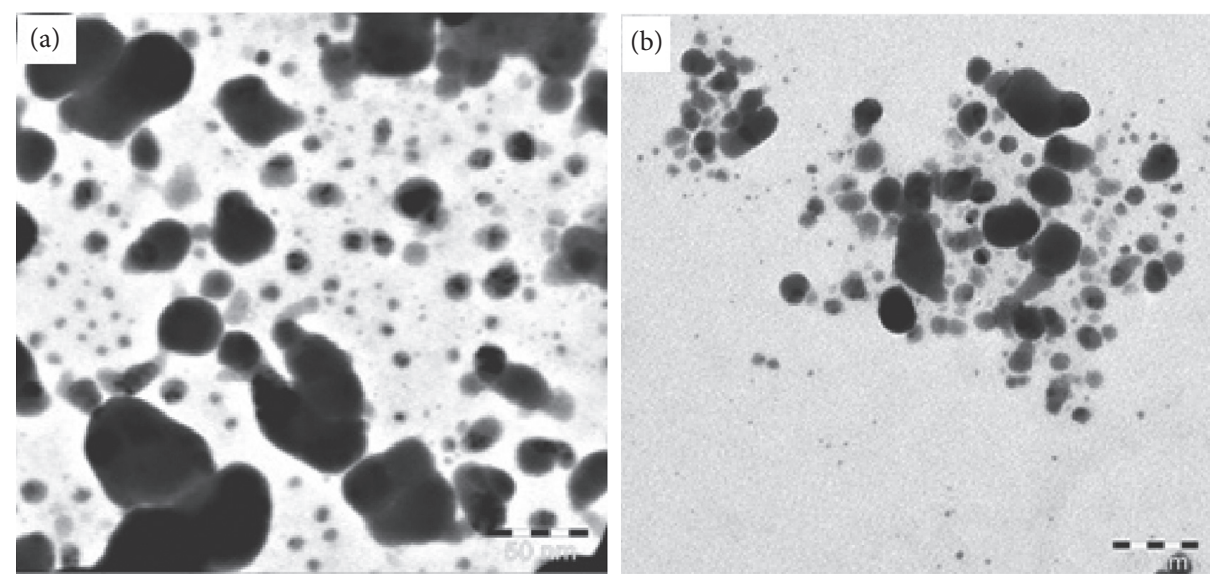

Figure 5: (a) High-resolution transmission electron microscopy micrographs of prepared AgNPs from Ch-TQ; (b) high-resolution transmission electron microscopy micrographs of prepared AgNPs from Ch-TAMPy.

TABle 1: Antimicrobial activity of Ch-TQ and Ch-TAMPy nanoparticles against the tested pathogenic microbes.

\begin{tabular}{|c|c|c|c|c|c|c|}
\hline \multirow{3}{*}{ Compd. } & \multirow{3}{*}{ Conc. $(\mu \mathrm{g})$} & \multicolumn{5}{|c|}{ Zone of inhibition $(\mathrm{mm})^{\dagger}$} \\
\hline & & \multicolumn{2}{|c|}{ Gram-negative } & \multicolumn{2}{|c|}{ Gram-positive } & \multirow[b]{2}{*}{ C. albicans } \\
\hline & & E. coli & P. aeruginosa & M. luteus & MRSA & \\
\hline \multirow{4}{*}{ Ch-TAMPy-AgNPs } & 10 & 14 & 14 & 18 & 18 & 14 \\
\hline & 20 & 18 & 18 & 22 & 20 & 20 \\
\hline & 30 & 20 & 20 & 26 & 22 & 22 \\
\hline & 40 & 22 & 22 & 30 & 24 & 24 \\
\hline \multirow{4}{*}{ Ch-TQ-AgNPs } & 10 & 12 & 12 & 16 & 16 & 12 \\
\hline & 20 & 14 & 14 & 18 & 18 & 12 \\
\hline & 30 & 16 & 16 & 20 & 20 & 14 \\
\hline & 40 & 18 & 18 & 23 & 22 & 14 \\
\hline
\end{tabular}

${ }^{\dagger}$ The initial size of the wells is $7 \mathrm{~mm}$.

\section{Conclusion}

The two modified chitosan-s-triazinyl-bis(2-aminomethylpyridine) (Ch-TAMPy) and chitosan-s-triazinylbis(8-oxyquinoline) (Ch-TQ) derivatives showed good thermal stability compared with chitosan itself as observed from TGA degradation. The two derivatives were used for preparation of AgNPs under ecofriendly conditions (water medium) without using any reducing agent or stabilizer with a particle size of AgNPs less than $10 \mathrm{~nm}$ as observed from TEM. Silver nanoparticles have good stability at room temperature for 5-6 months which indicated that chitosan protects the AgNPs from agglomeration. The AgNPs immobilized with new modified chitosan (Ch-TAMPy and Ch-TQ) showed high antimicrobial activity against the tested Gram-positive bacteria (M. luteus and MRSA), lowest activity against C. albicans, and had a moderate activity against Gram-negative bacteria ( $E$. coli and $P$. aeruginosa). These results could be useful for development of biocompatible polymers with antimicrobial activity and can be used in the textile industry or in wound dressing.

\section{Data Availability}

The data used in this study to support the findings are included in the article and supporting information.

\section{Conflicts of Interest}

The authors declare no conflicts of interest.

\section{Acknowledgments}

The authors would like to extend their sincere appreciation to the Deanship of Scientific Research at King Saud University for providing funding to the research group no. RG1441-365, Saudi Arabia.

\section{Supplementary Materials}

Method S1: methodology of the antibacterial activity of the modified chitosan-AgNPs against the tested organism; Figure S1: ninhydrin test; Figure S2: preparation of AgNPs using modified chitosan Ch-TQ; Figure S3: UV-Vis spectra of silver nanoparticle solutions; Figures S4A and S4B: energy-dispersive X-ray spectra (EDX) of the prepared silver nanoparticles from Ch-TQ and Ch-TAMPy; Figures S5A and S5B: AgNPs from Ch-TQ and Ch-TAMPy and their particle diagram; Table S1: information derived from TG curves for thermal degradation of copolymers; Table S2: $2 \theta$ and $d$ spacing for AgNPs calculated and observed by Ch-TQ and Ch-TAMPy; Table S3: lattice parameters, unit cell volume, and space group for AgNPs by Ch-TQ and ChTAMPy; and Table S4: average grain size $(D)$, dislocation 
density $(\delta)$, and the strain $(\varepsilon)$ of the AgNPs. (Supplementary Materials)

\section{References}

[1] M. Mazur, "Electrochemically prepared silver nanoflakes and nanowires," Electrochemistry Communications, vol. 6, no. 4, pp. 400-403, 2004.

[2] L. Feng, C. Zhang, G. Gao, and D. Cui, "Facile synthesis of hollow $\mathrm{Cu}_{2} \mathrm{O}$ octahedral and spherical nanocrystals and their morphology-dependent photocatalytic properties," Nanoscale Research Letters, vol. 7, no. 1, pp. 276-280, 2012.

[3] A. Pal, S. Shah, and S. Devi, "Preparation of silver, gold and silver-gold bimetallic nanoparticles in w/o microemulsion containing TritonX-100," Colloids and Surfaces A: Physicochemical and Engineering Aspects, vol. 302, no. 1-3, pp. 483-487, 2007.

[4] M. J. Rosemary and T. Pradeep, "Solvothermal synthesis of silver nanoparticles from thiolates," Journal of Colloid and Interface Science, vol. 268, no. 1, pp. 81-84, 2003.

[5] Y. Xie, R. Ye, and H. Liu, "Synthesis of silver nanoparticles in reverse micelles stabilized by natural biosurfactant," Colloids and Surfaces A: Physicochemical and Engineering Aspects, vol. 279, no. 1-3, pp. 175-178, 2006.

[6] S. Schultz, D. R. Smith, J. J. Mock, and D. A. Schultz, "Singletarget molecule detection with nonbleaching multicolor optical immunolabels," Proceedings of the National Academy of Sciences, vol. 97, no. 3, pp. 996-1001, 2000.

[7] T. A. Taton, C. Mirkin, and R. Letsinger, "Scanometric DNA array detection with nanoparticle probes," Science, vol. 289, no. 5485 , pp. $1757-1760,2000$.

[8] J. Yguerabide and E. E. Yguerabide, "Light-scattering submicroscopic particles as highly fluorescent analogs and their use as tracer labels in clinical and biological applications," Analytical Biochemistry, vol. 262, no. 2, pp. 157-176, 1998.

[9] M. Alavi and M. Rai, "Recent progress in nanoformulations of silver nanoparticles with cellulose, chitosan, and alginic acid biopolymers for antibacterial applications," Applied Microbiology and Biotechnology, vol. 103, no. 21-22, pp. 8669-8676, 2019.

[10] M. Alavi and M. Rai, "Recent advances in antibacterial applications of metal nanoparticles (MNPs) and metal nanocomposites (MNCs) against multidrug-resistant (MDR) bacteria," Expert Review of Anti-infective Therapy, vol. 17, no. 6, pp. 419-428, 2019.

[11] K. Kalwar and D. Shan, "Antimicrobial effect of silver nanoparticles (AgNPs) and their mechanism - a mini review," Micro \& Nano Letters, vol. 13, no. 3, pp. 277-280, 2018.

[12] T. K. Sau, A. Pal, N. R. Jana, Z. L. Wang, and T. Pal, "Size controlled synthesis of gold nanoparticles using phytochemically prepared seed particles," Journal of Nanoparticle Research, vol. 3, no. 4, pp. 257-261, 2001.

[13] K. N. Thakkar, S. S. Mhatre, and R. Y. Parikh, "Biological synthesis of metallic nanoparticles," Nanomedicine: Nanotechnology, Biology and Medicine, vol. 6, no. 2, pp. 257-262, 2010.

[14] H. Bar, D. K. Bhui, G. P. Sahoo, P. Sarkar, S. P. De, and A. Misra, "Green synthesis of silver nanoparticles using latex of Jatropha curcas," Colloids and Surfaces A: Physicochemical and Engineering Aspects, vol. 339, no. 1-3, pp. 134-139, 2009.

[15] A. Frattine, N. Pellegri, D. Nicastro, and O. de Sanctis, "Preparation of amine coated silver nanoparticles using triethylenetetraamine," Material Chemistry and Physics, vol. 94, pp. 148-152, 2005.
[16] M. Bin Ahmad, J. J. Lim, K. Shameli, N. A. Ibrahim, M. Y. Tay, and B. W. Chieng, "Antibacterial activity of silver bionanocomposites synthesized by chemical reduction route," Chemistry Central Journal, vol. 6, pp. 101-110, 2012.

[17] Z. Khan, S. A. Al-Thabaiti, E. H. El-Mossalamy, and A. Y. Obaid, "Studies on the kinetics of growth of silver nanoparticles in different surfactant solutions," Colloids and Surfaces B: Biointerfaces, vol. 73, no. 2, pp. 284-288, 2009.

[18] H. I. Won, H. Nersisyan, C. W. Won, J.-M. Lee, and J.-S. Hwang, "Preparation of porous silver particles using ammonium formate and its formation mechanism," Chemical Engineering Journal, vol. 156, no. 2, pp. 459-464, 2010.

[19] I. Pastoriza and L. M. Liz-Marzan, "Reduction of silver nanoparticles in DMF. Formation of monolayers and stable colloids," Pure and Applied Chemistry, vol. 72, no. 1-2, pp. 83-90, 2000.

[20] S. D. Solomon, M. Bahadory, A. V. Jeyarajasingam, S. A. Rutkowsky, and C. Boritz, "Synthesis and study of silver nanoparticles," Journal of Chemical Education, vol. 84, no. 2, pp. 322-325, 2007.

[21] S. Jaiswal, B. Duffy, A. K. Jaiswal, N. Stobie, and P. McHale, "Enhancement of the antibacterial properties of silver nanoparticles using $\beta$-cyclodextrin as a capping agent," International Journal of Antimicrobial Agents, vol. 36, no. 3, pp. 280-283, 2010.

[22] S. Singh, P. Patel, S. Jaiswal, A. A. Prabhune, C. V. Ramana, and B. L. V. Prasad, "A direct method for the preparation of glycolipid-metal nanoparticle conjugates: sophorolipids as reducing and capping agents for the synthesis of water redispersible silver nanoparticles and their antibacterial activity," New Journal of Chemistry, vol. 33, no. 3, pp. 646-652, 2009.

[23] J. K. Schluesener and H. J. Schluesener, "Nanosilver: application and novel aspects of toxicology," Archives of Toxicology, vol. 87, no. 4, pp. 569-576, 2013.

[24] S. Poulose, T. Panda, P. P. Nair, and T. Théodore, "Biosynthesis of silver nanoparticles," Journal of Nanoscience and Nanotechnology, vol. 14, no. 2, pp. 2038-2049, 2014.

[25] J. Y. Song and B. S. Kim, "Rapid biological synthesis of silver nanoparticles using plant leaf extracts," Bioprocess and Biosystems Engineering, vol. 32, no. 1, pp. 79-84, 2009.

[26] N. J. Reddy, D. Nagoor Vali, M. Rani, and S. S. Rani, "Evaluation of antioxidant, antibacterial and cytotoxic effects of green synthesized silver nanoparticles by Piper longum fruit," Materials Science and Engineering: C, vol. 34, pp. 115-122, 2014.

[27] E. C. N. Lopes, K. S. Sousa, and C. Airoldi, "Chitosan-cyanuric chloride intermediary as a source to incorporate moleculesthermodynamic data of copper/biopolymer interactions," Thermochimica Acta, vol. 483, no. 1-2, pp. 21-28, 2009.

[28] M. N. V. Ravi Kumar, "A review of chitin and chitosan applications," Reactive and Functional Polymers, vol. 46, no. 1, pp. 1-27, 2000.

[29] E. Guibal, "Heterogeneous catalysis on chitosan-based materials: a review," Progress in Polymer Science, vol. 30, no. 1, pp. 71-109, 2005.

[30] K. Erol, M. Bolat, D. Tatar, C. Nigiz, and D. A. Köse, "Synthesis, characterization and antibacterial application of silver nanoparticle embedded composite cryogels," Journal of Molecular Structure, vol. 1200, Article ID 127060, 2020.

[31] X. Liu, W. Xia, Q. Jiang, Y. Xu, and P. Yu, "Effect of kojic acidgrafted-chitosan oligosaccharides as a novel antibacterial agent on cell membrane of gram-positive and gram-negative 
bacteria," Journal of Bioscience and Bioengineering, vol. 120, no. 3, pp. 335-339, 2015.

[32] W. Xia, P. Liu, J. Zhang, and J. Chen, "Biological activities of chitosan and chitooligosaccharides," Food Hydrocolloids, vol. 25, no. 2, pp. 170-179, 2011.

[33] X. Liu, W. Xia, Q. Jiang, Y. Xu, and P. Yu, "Synthesis, characterization, and antimicrobial activity of kojic acid grafted chitosan oligosaccharide," Journal of Agricultural and Food Chemistry, vol. 62, no. 1, pp. 297-303, 2013.

[34] A. Soozanipour, A. Taheri-Kafrani, M. Barkhori, and M. Nasrollahzadeh, "Preparation of a stable and robust nanobiocatalyst by efficiently immobilizing of pectinase onto cyanuric chloride-functionalized chitosan grafted magnetic nanoparticles," Journal of Colloid and Interface Science, vol. 536, pp. 261-270, 2019.

[35] H. Minamisawa, K. Murashima, M. Minamisawa, N. Arai, and T. Okutani, "Determination of indium by graphite furnace atomic absorption spectrometry after coprecipitation with chitosan," Analytical Sciences, vol. 19, no. 3, pp. 401-404, 2003.

[36] H. Minamisawa, N. Arai, and T. Okutani, "Electrothermal atomic absorption spectrometric determination of copper(II) using a tungsten metal furnace after preconcentration onto chitosan," Analytical Sciences, vol. 15, no. 3, pp. 269-275, 1999.

[37] Y. Gao, K. Oshita, K.-H. Lee, M. Oshima, and S. Motomizu, "Development of column-pretreatment chelating resins for matrix elimination/multi-element determination by inductively coupled plasma-mass spectrometry," The Analyst, vol. 127, no. 12, pp. 1713-1719, 2002.

[38] R. Bassi, S. O. Prasher, and B. K. Simpson, "Effects of organic acids on the adsorption of heavy metal ions by chitosan flakes," Journal of Environmental Science and Health, Part A, vol. 34, no. 2, pp. 289-294, 1999.

[39] M. L. Wang, S. H. Qian, S. B. Mo, G. Q. Huang, and H. Y. Wang, "Application of crosslinked chitosan in the analysis of butyl Tin speciation," Journal of Applied Polymer Science, vol. 93, no. 3, pp. 1326-1328, 2004.

[40] S. Nishimura, O. Kohgo, K. Kurita, and H. Kuzuhara, "Chemospecific manipulations of a rigid polysaccharide: syntheses of novel chitosan derivatives with excellent solubility in common organic solvents by regioselective chemical modifications," Macromolecules, vol. 24, no. 17, pp. 47454748, 1991.

[41] P. K. Datta, P. S. Basu, and T. K. Datta, "Interaction of Vicia faba lectin with methyl $\alpha$-d-mannopyranoside, investigated by ultraviolet difference spectroscopy," Preparative Biochemistry, vol. 14, pp. 373-387, 1984.

[42] S. M. Hudson and D. W. Jenkins, "Chitin and chitosan," in EPST, H. F. Mark, Ed., vol. 1, pp. 569-580, Wiley, New York, NY, USA, 3rd edition, 2003.

[43] A. Martins, S. Facchi, H. Follmann, A. Pereira, A. Rubira, and E. Muniz, "Antimicrobial activity of chitosan derivatives containing $N$-quaternized moieties in its backbone: a review," International Journal of Molecular Sciences, vol. 15, no. 11, pp. 20800-20832, 2014.

[44] A. El-Faham, K. Dahlous, Z. AL Othman, H. Al-Lohedan, and G. El-Mahdy, "sym-Trisubstituted 1,3,5-triazine derivatives as promising organic corrosion inhibitors for steel in acidic solution," Molecules, vol. 21, no. 4, pp. 436-447, 2016.

[45] A. W. Bauer, W. M. M. Kirby, J. C. Sherris, and M. Turck, "Antibiotic susceptibility testing by a standardized single disk method," American Journal of Clinical Pathology, vol. 45, no. 4, pp. 493-496, 1966.
[46] C. Krishnaraj, E. G. Jagan, S. Rajasekar, P. Selvakumar, P. T. Kalaichelvan, and N. Mohan, "Synthesis of silver nanoparticles using Acalypha indica leaf extracts and its antibacterial activity against water borne pathogens," Colloids and Surfaces B: Biointerfaces, vol. 76, no. 1, pp. 50-56, 2010.

[47] M. M. Ganesh Babu and P. Gunasekaran, "Production and structural characterization of crystalline silver nanoparticles from Bacillus cereus isolate," Colloids and Surfaces B: Biointerfaces, vol. 74, no. 1, pp. 191-195, 2009.

[48] D. Wei, W. Sun, W. Qian, Y. Ye, and X. Ma, “The synthesis of chitosan-based silver nanoparticles and their antibacterial activity," Carbohydrate Research, vol. 344, no. 17, pp. 2375-2382, 2009.

[49] N. Q. Hien, D. V. Phu, N. N. Duy et al., "Influence of chitosan binder on the adhesion of silver nanoparticles on cotton fabric and evaluation of antibacterial activity," Advances in Nanoparticles, vol. 4, no. 4, pp. 98-106, 2015.

[50] P. Prema and S. Thangapandiyan, "A Comparative in vitro cytotoxicity study of colloidal silver and chitosan stabilized silver nanoparticles on MCF-7 and HEPG2," European Journal of Biomedical and Pharmaceutical Sciences, vol. 2, pp. 855-870, 2015.

[51] T. Elavazhagan and T. Elavazhagan, "Memecylon edule leaf extract mediated green synthesis of silver and gold nanoparticles," International Journal of Nanomedicine, vol. 6, pp. 1265-1278, 2011.

[52] C. Liu, X. Yang, H. Yuan, Z. Zhou, and D. Xiao, "Preparation of silver nanoparticle and its application to the determination of ct-DNA," Sensors, vol. 7, no. 5, pp. 708-718, 2007.

[53] K. Jyoti, M. Baunthiyal, and A. Singh, "Characterization of silver nanoparticles synthesized using Urtica dioica Linn. Leaves and their synergistic effects with antibiotics," Journal of Radiation Research and Applied Sciences, vol. 9, no. 3, pp. 217-227, 2016.

[54] N. B. Patel, S. N. Agravat, and F. M. Shaikh, "Synthesis and antimicrobial activity of new pyridine derivatives-I," Medicinal Chemistry Research, vol. 20, no. 7, pp. 1033-1041, 2011.

[55] M. Bankova, N. Manolova, N. Markova, T. Radoucheva, K. Dilova, and I. Rashkov, "Copolymers of 5-chloror-8-quinolinyl acrylate and acrylamide-synthesis, hydrolysis behavior and antibacterial activity," European Polymer Journal, vol. 34, pp. 247-253, 1998.

[56] M. Khan, S. T. Khan, M. Khan et al., "Antibacterial properties of silver nanoparticles synthesized using Pulicaria glutinosa plant extract as a green bioreductant," International Journal of Nanomedicine, vol. 9, pp. 3551-3565, 2014. 